\title{
Diseño y validación de un test de evaluación del potencial en emprendimiento social
}

\author{
May Portuguez Castro ${ }^{1}$, Jaime Ricardo Valenzuela González ${ }^{2}$ y Claudia Navarro Corona ${ }^{3}$
}

Recibido: 30 de noviembre de 2017 / Aceptado: 12 de abril de 2018

Resumen. El emprendedor social se reconoce como una persona que es capaz de determinar oportunidades de negocio, a la vez que soluciona un problema que afecta a otras personas. En la literatura se encuentran pocos estudios que estén enfocados hacia este tipo de emprendedor, ya que generalmente se realizan investigaciones sobre los emprendedores en general, sin reconocer las características de los emprendedores sociales. Este estudio valida un instrumento diseñado para medir el potencial en emprendimiento social que tiene una persona. La finalidad del instrumento es que funcione como una herramienta para quienes realizan labores de incubación de empresas, así como de capacitación para los emprendedores sociales; también puede ser utilizado dentro de organizaciones que manejen programas de responsabilidad social, como las cooperativas, con el fin de que tomen en cuenta las necesidades del emprendedor a la hora de diseñar estrategias de desarrollo para estas personas y sus emprendimientos. Además, para que los mismos emprendedores puedan determinar su potencial en el desarrollo de estas empresas.

Palabras clave: Emprendedor social; Pasión hacia el emprendimiento; Sustentabilidad; Empresa social; Potencial emprendedor.

Claves Econlit: M14; L26; I29.

\section{[en] Development and validation of the assessment test of social entrepreneurial potential}

\begin{abstract}
A social entrepreneur is a person capable to recognize business opportunities and to solve problems that affect other people. The few studies available in the literature about this kind of entrepreneur, make necessary to develop research related to identify their characteristics. This study aims to validate an instrument to measure the social entrepreneurial potential. The purpose of the Assessment Test of Entrepreneurial Potential is to serve as a tool that helps business incubators, training programs designers, and it can also be used within programs that manage social responsibility programs, such as cooperatives, to know the entrepreneur needs. Moreover, the instrument allows entrepreneurs to identify their entrepreneurial potential to develop their social entrepreneurial skills.
\end{abstract}

Keywords: Social entrepreneur; Passion for entrepreneurship; Sustainability; Social business; Entrepreneurial potential.

1 Tecnológico de Monterrey, México

Dirección de correo electrónico: mportuguez1@ gmail.com

2 Tecnológico de Monterrey, México

Dirección de correo electrónico: jrvg@itesm.mx

3 Tecnológico de Monterrey, México

Dirección de correo electrónico: c.navarrocorona@gmail.com 
Sumario. 1. Introducción. 2. Revisión de la literatura: definición conceptual. 3. Método. 4. Resultados. 5. Conclusiones. 6. Referencias bibliográficas.

Cómo citar: Portuguez Castro, M., Valenzuela González, J.R. y Navarro Corona, C. (2018) Diseño y validación de un test de evaluación del potencial en emprendimiento social. REVESCO. Revista de Estudios Cooperativos, Segundo Cuatrimestre, No 128, pp. 192-211. DOI: 10.5209/REVE.60207.

\section{Introducción}

El emprendimiento social se define como la iniciativa de un empresario que tiene una visión social (Yunus, 2010). Esta visión puede ser no económica, de caridad, o de negocio sin ganancia personal, aunque también incluye, las actividades que buscan utilidades y las que están incluidas en una organización sin ánimo de lucro. El emprendimiento social tiene el interés de fortalecer una economía dirigida a resolver aquellos problemas que no son solventados por el mercado, el gobierno o el sector voluntario (Brinckerhoff, 2000). Estas características del emprendimiento social también están presentes en organizaciones como las cooperativas, las cuales tienen un alto componente de responsabilidad, pues tienen una preocupación por buscar alternativas a los problemas de la sociedad (Melián Navarro y Campos Climent, 2010).

La exigencia de responsabilidad social que tienen las empresas hace que la actividad económica esté orientada, no solo a la generación de ganancias, sino también a un compromiso en pro de la sustentabilidad, la mejora de las partes involucradas en las organizaciones y un efecto hacia afuera de la empresa, donde la comunidad pueda verse beneficiada (Iturrioz y Dopacio, 2009). Además, este tipo de emprendimiento se considera como una herramienta que puede ayudar a dinamizar las economías, especialmente en tiempos de crisis (Benito Hernández, 2009).

El emprendimiento social se relaciona con personas con ciertas características que les faciliten desarrollar esta tarea con éxito. Según Surie (2017), un emprendedor social es la persona que busca generar un valor para la comunidad, trabaja como un agente de cambio y que a la vez trata de beneficiar a la sociedad en problemas específicos. Debido a las particularidades de estos emprendimientos, es que en este estudio se definieron las características que debe tener la persona que lo desarrolle, con la finalidad de validar un instrumento que permita determinar el potencial para realizarlo.

Distintos investigadores en el tema del emprendimiento han coincidido en que los emprendedores sociales poseen características específicas que resultan clave para el éxito en la tarea. Si bien estas características son variadas debido a que el emprendedor social busca los objetivos empresariales para alcanzar un bien común, se buscaron características que se consideran relevantes para determinar si la persona tiene un perfil que le permita iniciar con una labor que favorece la combinación de lo social y lo económico (Navarro y Climent, 2010). Entre las características identificadas se encuentran: (1) la pasión por el emprendimiento, como un factor de motivación hacia las situaciones difíciles (Vallerand, 2010; Baum y Locke, 2004; Cardon, Glauser y Murnieks, 2017), (2) la orientación a la sustentabilidad, por su interés en la creación y explotación de nuevos bienes y 
servicios, orientados al bienestar ambiental y social (Hossain, Saleh y Drennan, 2017, DiVito y Bohnsack, 2017) y (3) la identificación de oportunidades, como paso fundamental para el desarrollo de ideas y una de las más importantes habilidades para su éxito (Sahai y Frese, 2017, Wry y York, 2017).

El objetivo principal de este estudio es determinar la confiabilidad y validez de un instrumento de evaluación de las características del emprendedor social, con el propósito de aportar una herramienta a las personas que participan en procesos de capacitación y de formación de empresas sociales a conocer las características de los emprendedores y poder desarrollar programas que potencien estas habilidades.

A continuación, se presenta una revisión de literatura, que servirá como guía para la creación del instrumento.

\section{Revisión de la literatura: definición conceptual}

En este apartado se definen teórica y operacionalmente, las subescalas que van a servir para medir el constructo de potencial de emprendimiento social. Primero se presenta la definición y posteriormente se exploran algunas herramientas usadas por otros autores para su medición.

\subsection{Pasión hacia el emprendimiento}

La pasión hacia el emprendimiento se define como aquellos sentimientos positivos e intensos que siente el emprendedor cuando participa en actividades empresariales (Biraglia y Karile, 2016). Dentro de un emprendimiento, estos sentimientos puedan enfocarse hacia las actividades que realizan dentro de la empresa, hacia los logros que obtiene a través del emprendimiento o hacia el desarrollo que ha creado (Cardon et al, 2017). Este concepto se ha estudiado en la psicología desde las teorías de liderazgo y se considera como una característica indispensable para los líderes exitosos en los negocios y que funciona como un factor de motivación.

Existen diferentes escalas que han sido diseñadas para establecer la pasión que una persona tiene hacia la actividad que realiza. A continuación, se describen algunas encontradas en la literatura y que se tomaron como base para realizar el instrumento:

\section{Escala de pasión por el trabajo}

Baum y Locke (2004), realizaron un estudio en el que determinaron la relación del amor al trabajo, con el éxito en las actividades de emprendimiento. Estos autores definieron el concepto de amor por el trabajo y lo midieron en términos de la emoción del amor, cariño o apego y tiempo invertido en realizar la actividad, por lo que consideran que la pasión puede ser observada en las largas horas invertidas por el emprendedor durante las fases de desarrollo de su proyecto.

Ejemplos de cómo se midió esta variable en este instrumento, fueron las respuestas a enunciados como: amo mi trabajo o, busco la forma de regresar a mi trabajo cuando estoy fuera de él. Con lo que se buscaba medir por medio de una escala Likert, si la persona estaba muy de acuerdo o muy en desacuerdo con el enunciado, para determinar su pasión por lo que realiza (Baum y Locke, 2004). 


\section{Escala de amor por el trabajo}

Algunos autores definen la pasión hacia el emprendimiento como la mayor cantidad de tiempo y esfuerzo que una persona emplea en alcanzar sus metas, o el amor que tiene hacia el trabajo que realiza (Gagné, 2014). De Clercq y Belausteguigoitia (2016), utilizaron estos enunciados para realizar la escala de amor por el trabajo: la mayor satisfacción de mi vida proviene de mi trabajo, yo cumplo con mucho trabajo porque amo trabajar, amo trabajar, algunas veces desearía estar trabajando cuando no lo estoy.

\section{Escala de la pasión}

Vallerand mencionado por Gagné (2014) describe que la pasión tiene una dualidad, la cual puede ser obsesiva o armoniosa. La pasión se considera armoniosa cuando existe una internalización de la actividad dentro de la persona, donde se comprende que es importante que se puede comprometer con lo que le gusta, pero sin afectar otras áreas de su vida personal. La persona que demuestra una pasión armoniosa tiene control sobre la actividad y puede decidir cuándo involucrarse o no en la misma, es una forma de pasión más autónoma que la obsesiva, aunque ambas tienen la misma cantidad de amor por lo que se realiza (Gagné, 2014).

Cuando una persona siente pasión por lo que hace, presenta las siguientes características: tiene emociones positivas hacia los conflictos, por lo que desarrollan mejores habilidades para la solución de problemas, mayor compromiso hacia el logro de metas y enfrentan mejor el estrés por su motivación intrínseca de triunfar (De Clercq y Belausteguigoitia, 2016).Vallerand (2010) desarrolló un instrumento para medir esta variable y que es conocido como Escala de la pasión, el cual consta de 16 ítems y los divide para cada tipo en armoniosa y obsesiva. Cada una es evaluada en una escala tipo Likert de 1 a 7 , desde no estar de acuerdo del todo hasta muy de acuerdo. Al medir la pasión con esta escala se puede determinar el grado de amor que se tiene hacia una actividad que se considera importante en la vida de la persona y a la cual se le va a dedicar mucho tiempo y energía.

\section{Versión española de la escala de la pasión por el trabajo}

Serrano-Fernández, Boada-Grau, Gil-Ripoll y Vigil Colet (2017) agregaron a la evaluación realizada por Vallerand algunos enunciados que también ayudan a medir la cantidad de tiempo empleada para una actividad. Este instrumento consulta, por ejemplo, si frecuentemente lleva trabajo a casa, el número de noches que se despierta pensando en cosas de trabajo, las veces que le dice a otras personas que debería estar trabajando, el número de citas que ha perdido por estar trabajando y las veces que no ha dedicado tiempo a actividades personales por estar trabajando, entre otras.

En este estudio se utilizarán enunciados de los autores antes mencionados, para elaborar un instrumento que pueda medir la pasión que tienen los participantes, determinada por el amor que tienen hacia esa actividad y la cantidad de tiempo y energía que empleen en realizarla. 


\subsection{Orientación a la sustentabilidad}

Uno de los elementos más importantes dentro de los emprendimientos sociales es el desarrollo de empresas sustentables y orientadas a la creación de soluciones que mejoren las condiciones de la población (Hossain et al, 2017). La sustentabilidad se define como cubrir las necesidades del presente sin comprometer la habilidad de las nuevas generaciones para cubrir sus propias necesidades (Roxas, 2012). Tener un comportamiento sustentable consiste en poder desarrollar soluciones a los retos de hoy tomando en cuenta que no afecten a las personas en el futuro (Kuckertz, y Wagner, 2010).

Como mencionan DiVito y Bohnsack (2017), la orientación a la sustentabilidad es la convicción que tiene el emprendedor de hacer que su negocio se desarrolle ecológicamente y sea socialmente responsable. Sin embargo, las características que requiere esta clase de emprendedor no son observables en todas las personas, ya que como indican Cohen y Winn mencionados por Kuckertz y Wagner (2010), estos emprendedores deben saber reconocer cuáles bienes y servicios pueden ser explotados y creados en el futuro. Además de conocer las consecuencias tanto económicas, sociales y ambientales que se pueden desarrollar.

Chen, Huand, Long y Li (2017), mencionan que algunos comportamientos que pueden ser observados los emprendedores sociales son: ahorrar agua y electricidad, reciclar, consumir productos amigables con el ambiente y persuadir a otros para proteger el ambiente. Algunos investigadores ya han trabajado con instrumentos que pueden medir este constructo y que son descritas a continuación.

\section{Escala de comportamiento ecológico general}

La escala de comportamiento ecológico general analiza 6 grandes dominios en los que se puede observar comportamientos relacionados con la orientación a la sustentabilidad (Boeve-de Pauw y Van Petegem, 2017). Algunos ítems utilizados en esta escala son: "Tengo un auto eficiente", "Reutilizo las bolsas del supermercado", "Compro productos amigables con el ambiente" (Kaiser, Scheuthle y Carabias, 2005).

\section{Orientación a la sustentabilidad}

Corral-Verdugo, Tapia y Ortiz-Valdez (2014), indican que la orientación a la sustentabilidad incluye comportamientos pro-ecológicos, altruismo, frugalidad y equidad. Los comportamientos pro-ecológicos son aquellas acciones que llevan a la conservación de los recursos naturales, como son: el reciclaje, conservación del agua, lectura de temas ambientales, persuadir a otros a cuidar el ambiente, entre otros (Herrera-Mendoza, 2016). El altruismo se define como las actitudes que van orientadas a ayudar a otros sin esperar obtener nada a cambio, e incluye acciones como la participación en actividades que mitiguen los efectos de fenómenos naturales, participen en la recaudación de fondos y atiendan las necesidades de personas afectadas (Corral-Verdugo et al, 2014).

La frugalidad según Tapia-Fonllem, Corral, Fraijo y Durón (2013), es lo contrario al consumismo, se refiere a disminuir el consumo de productos con el fin de disminuir el impacto que se tiene sobre los recursos naturales. Implica según estos autores, todas las acciones que se realizan para reducir el consumo, así como 
el tipo de cosas que se adquieren evitando el desperdicio. Por último, la equidad corresponde a buscar la justa distribución de los recursos entre las personas, permitir los mismos beneficios entre hombres y mujeres y el trato igualitario entre las minorías y personas de todas las clases sociales (Tapia-Fonllem, Fraijo-Sing, Corral Verdugo y Ortiz Valdez, 2017).

Los enunciados anteriores van a incluirse en el instrumento que se va a diseñar en este estudio como parte del constructo de potencial emprendedor, analizando la conducta pro-ecológica y las actitudes del emprendedor hacia la frugalidad, altruismo y equidad para conocer si cuenta con orientación a la sustentabilidad.

\subsection{Identificación de oportunidades}

Vogel (2016) menciona que un concepto importante dentro del emprendimiento social es reconocer la oportunidad donde desarrollar la actividad. Este conocimiento comprende el reconocimiento y la interpretación de la información que recibe el emprendedor para determinar si valora su desarrollo. Dos elementos importantes dentro de la identificación de oportunidades son: el conocimiento que se tenga sobre el área a desarrollar, pues a mayor conocimiento, el emprendedor puede determinar mejor su viabilidad y las relaciones sociales con las que cuente y así dirigir su atención hacia oportunidades en su entorno (Wry y York, 2017).

Las oportunidades de emprendimiento salen de las imperfecciones del mercado y es allí donde el emprendedor social reconoce cuáles son las que puede desarrollar y realizar como un emprendimiento (Martin y Binder, 2015). Kliksberg (2011) explica que el emprendedor social identifica problemas de exclusión y marginación y busca oportunidades para cambiar estas realidades, para así generar un nuevo equilibrio que garantice a estos grupos mejores condiciones de vida y un beneficio a la comunidad. Esta habilidad hace que el emprendedor social se convierta en un administrador que es capaz de combinar eficientemente los recursos para aprovechar las oportunidades que se le presentan (Ciruela, Cuadrado y Angulo, 2016)

Álvarez y Barney (2014) mencionan que existen tres tipos de oportunidades: el autoempleo en la cual el emprendedor realiza una actividad para satisfacer una necesidad, ésta se considera limitada ya que no puede ser escalable ni generar más empleos, únicamente para la persona que detecta la necesidad y pone en práctica una solución. El otro tipo de oportunidad que mencionan estos autores es el descubrimiento de oportunidades, el cual está formado por condiciones externas al mercado como pueden ser cambios en la tecnología, en políticas de gobierno o en la población, entre otras. En este caso el emprendedor detecta la necesidad antes que alguien más y propone una solución que puede ser imitada y es escalable por lo que genera otras oportunidades (Álvarez y Barney, 2014).

Por último, en la creación de oportunidades el factor humano es lo más importante ya que es el emprendedor quien va a crear las oportunidades que van a surgir gracias a su potencial emprendedor (Welter, Mauer y Wuebker, 2016). González, Husted y Ainer (2017) mencionan que son las personas que utilizan los recursos que tienen a mano para solventar los problemas que se les presentan, lo que implica un bajo temor a fallar y un buen uso de los recursos con que cuenta. 
Para definir si la persona es capaz de identificar oportunidades se mencionan dos instrumentos que sirvieron de referencia para este estudio:

\section{Descubrimiento y creación de oportunidades en el emprendimiento social}

Para medir este constructo González et al (2017) aplicaron un instrumento que toma en cuenta tanto el descubrimiento de emprendimientos sociales, como su creación. Con esta herramienta se midieron las siguientes variables para determinar en el emprendedor el descubrimiento de oportunidades:

- Alerta empresarial: Es cuando el emprendedor social analiza e interpreta un problema social que atrae su atención e inicia una búsqueda de información sobre las posibles soluciones al problema que ya han sido utilizadas. Utiliza enunciados como: yo busco soluciones para resolver los problemas o yo busco cualquier solución que haya sido tomada en otros lugares.

- Percepción del ambiente: Se refiere al conocimiento que tiene el emprendedor de la realidad que lo rodea. Entre mejor conozca su entorno será más fácil encontrar soluciones a los problemas y determinar las necesidades que existen. Utiliza enunciados como: Yo creo que mi percepción me permite encontrar soluciones a los problemas, mi percepción sobre el problema es buena.

- Redes sociales del emprendedor: Comprende el grupo de personas que tiene el emprendedor que no son personas tan cercanas, pero que sí son personas que le pueden aportar información sobre las necesidades que existen y poder encontrar mayores oportunidades. Los enunciados que se utilizan son: Conozco personas que están trabajando en desarrollar soluciones para problemas en los que estoy interesado o tengo una amplia red social que me permite tener información para solucionar los problemas.

\section{Tarea de identificación de oportunidades}

Otra forma de medir la identificación de oportunidades es la utilizada por Sahai y Frese (2017), en la cual se utilizan el conocimiento previo que tiene el emprendedor para identificar su capacidad de desarrollar nuevas ideas. En este estudio los autores presentaron a los participantes una información con un problema a resolver y se les pidió contestar con todas las ideas de negocio que pudieran, los resultados se midieron de dos formas: por la cantidad de oportunidades identificadas y por lo innovadoras que fueron estas ideas. En el instrumento diseñado para este estudio, se incluyó un caso en el que la persona pudo comentar cuáles oportunidades de solución observaba, y así determinar si lograba identificar soluciones al problema. A continuación, se presenta el método seguido para validar el instrumento.

\section{Método}

El test de evaluación del potencial en emprendimiento social presenta un conjunto de reactivos que buscan medir actitudes y comportamientos relacionados con el potencial para realizar actividades de emprendimiento social entendido en los 
términos centrales de Navarro y Climent (2010), Vallerand (2010), Baum y Locke (2004) Cardon y otros (2017), Hossain y otros (2017) y el resto de los autores.

\subsection{Participantes}

El instrumento fue aplicado a una muestra de 79 emprendedores que forman parte de una red de incubadoras de una universidad ubicada en el norte de México. Los encargados de las incubadoras fueron invitados a participar y se les pidió que enviaran por correo electrónico el instrumento a sus afiliados. La selección de la muestra es no aleatoria, debido a que participaron las personas que decidieron responder el cuestionario, quienes enviaron sus resultados por medio del mismo sistema de recolección de datos.

Los participantes son personas que desarrollan un emprendimiento o tienen una idea de negocio para desarrollar. En esta muestra se encontró que el $59.5 \%$ son hombres (47) y el $40.5 \%$ son mujeres (32). El grado académico más común es el universitario completo (56.8\% de la muestra), un $10.8 \%$ tienen universidad incompleta y el 3.6\% restante, secundaria completa. La edad de los participantes está entre 19 y 61 años, con un promedio de 33.67 años y una desviación estándar de 10.42 años. En cuanto al tiempo de tener el emprendimiento, el mínimo es de 1 mes y el máximo es de 18 años, con un promedio de 3,4 años y una desviación estándar de 4 años.

\subsection{Procedimiento}

El test de evaluación del potencial en emprendimiento social fue creado como un formulario electrónico y se compartió, junto con las condiciones de participación, con los encargados de las incubadoras. Éstos enviaron el instrumento por correo electrónico a los participantes, quienes lo completaron y enviaron sus resultados por la misma vía.

\subsection{Descripción del test}

El test consiste en una escala tipo Likert, que consta de 30 ítems distribuidos en ocho subescalas, relacionados con tres dimensiones pertenecientes al emprendimiento social: pasión hacia el emprendimiento (12 ítems), orientación hacia la sustentabilidad (14 ítems) e identificación de oportunidades (3 ítems y una pregunta abierta). La posible respuesta de cada enunciado tiene un puntaje que va de: siempre (5 puntos); casi siempre (4 puntos); algunas veces (3 puntos); casi nunca ( 2 puntos) y nunca (1 punto). El puntaje mínimo global es 30 puntos y el máximo es de 155 puntos.

El cuestionario está dividido en tres partes: la primera incluye datos personales del participante como: edad, género, dirección, grado académico e información sobre su emprendimiento, tiempo de realizarlo y tipo. La segunda parte consiste en la presentación de un caso ficticio donde el participante debe dar soluciones a un problema planteado, y el resto del instrumento contiene los diferentes reactivos que se van a medir, para cada una de las subescalas que definen las dimensiones que 
conforman el potencial de emprendimiento social. El test se encuentra en el Anexo 1.

Una vez que se aplica el test, las mismas personas pueden calcular sus resultados. El instrumento se califica utilizando la puntuación para cada uno de los reactivos, según la subescala que se esté evaluando y luego convirtiendo esos puntos en un porcentaje. Una vez convertidos los porcentajes, éstos se suman para definir el potencial de emprendimiento social de cada participante, si es cercano a $100 \%$, el potencial se considera alto. Para realizar esta calificación, se propone utilizar la forma de evaluación que se presenta en la Tabla 1, donde cada número corresponde al reactivo dentro del test que pretende medir esa subescala.

Tabla. 1. Potencial de emprendimiento social.

\begin{tabular}{|c|c|c|c|c|c|c|c|c|}
\hline \multicolumn{3}{|c|}{$\begin{array}{l}\text { Pasión hacia el } \\
\text { emprendimiento }\end{array}$} & \multicolumn{4}{|c|}{ Orientación a la sustentabilidad } & \multicolumn{2}{|c|}{$\begin{array}{l}\text { Identificación de } \\
\text { oportunidades }\end{array}$} \\
\hline $\begin{array}{l}\text { Apego } \\
\text { hacia la } \\
\text { actividad }\end{array}$ & $\begin{array}{l}\text { Pasión } \\
\text { armoniosa }\end{array}$ & $\begin{array}{l}\text { Dedicación } \\
\text { hacia el } \\
\text { emprendi- } \\
\text { miento }\end{array}$ & $\begin{array}{l}\text { Conducta } \\
\text { pro- } \\
\text { ecológica }\end{array}$ & Frugalidad & Altruismo & Equidad & $\begin{array}{l}\text { Ítems } \\
\text { del } \\
\text { cuestic } \\
\text { nario }\end{array}$ & $\begin{array}{l}\text { Pregunta } \\
\text { abierta }\end{array}$ \\
\hline 1 & 16 & 4 & 5 & 3 & 7 & 20 & 2 & $\begin{array}{l}\text { Ninguna } \\
\text { respuesta } \\
0 \text { pts. }\end{array}$ \\
\hline 6 & 23 & 11 & 10 & 18 & 12 & 21 & 9 & $\begin{array}{l}\text { De una a } \\
\text { 3:5puntos }\end{array}$ \\
\hline 8 & 27 & 14 & 15 & 28 & 13 & 26 & $7^{1}$ & $\begin{array}{l}\text { Más de 4: } \\
10 \text { pts. }\end{array}$ \\
\hline 24 & 29 & 30 & 19 & & 22 & & & \\
\hline \multicolumn{3}{|c|}{$\begin{array}{l}\mathrm{PE}=(\text { Total de puntos } / 60) \\
* 30 \%\end{array}$} & \multicolumn{4}{|c|}{$\mathrm{OS}=($ Total de puntos $/ 80) * 50 \%$} & \multicolumn{2}{|c|}{$\begin{array}{l}\mathrm{IO}=(\text { Total de } \\
\text { puntos } / 25) * 20 \%\end{array}$} \\
\hline
\end{tabular}

Potencial de Emprendimiento Social= $\mathrm{PE}+\mathrm{OS}+\mathrm{IO}$

Los datos que se presentan a continuación describen el perfil de emprendimiento social encontrado en los participantes de esta muestra, así como el análisis psicométrico de los datos de las características del instrumento utilizado.

\section{Resultados}

En la primera sección se muestran los resultados obtenidos por la muestra que respondió el cuestionario y en la segunda parte, el análisis psicométrico del instrumento. 


\subsection{Características de la muestra estudiada}

Los resultados para cada de las subescalas medidas en el test de potencial de emprendimiento social se muestran en la Tabla 2. Para analizar los resultados obtenidos se compara la media del grupo con la media esperada (que resulta de sumar el mínimo y máximo posible y dividirlo entre dos). Se puede observar que los valores para las escalas de apego a la actividad, dedicación y frugalidad estuvieron cercanos a la media esperada. En el caso de la pasión al emprendimiento, la conducta pro-ecológica y el altruismo, los resultados fueron más altos que la media, pero no se aproximaron al valor máximo. El resultado más alto para esta población se dio en el de orientación a la sustentabilidad, lo que indicó que esta variable fue la que más estaba presente en estos emprendedores.

Tabla. 2. Características de la muestra por subescala

\begin{tabular}{|c|c|c|c|c|c|c|}
\hline Subescala & Mediana & Media & Mínimo & Máximo & $\begin{array}{l}\text { Media } \\
\text { esperada }\end{array}$ & $\begin{array}{l}\text { Desviación } \\
\text { estándar }\end{array}$ \\
\hline $\begin{array}{l}\text { Apego hacia la } \\
\text { actividad }\end{array}$ & 16 & 15.89 & 10 & 20 & 15 & 2.587 \\
\hline Pasión armoniosa & 17 & 16.57 & 8 & 20 & 14 & 2.763 \\
\hline Dedicación & 12 & 12.10 & 7 & 18 & 12.5 & 3.112 \\
\hline $\begin{array}{l}\text { Pasión hacia el } \\
\text { emprendimiento }\end{array}$ & 45 & 44.56 & 27 & 57 & 42 & 6.590 \\
\hline $\begin{array}{l}\text { Conducta pro- } \\
\text { ecológica }\end{array}$ & 15 & 14.18 & 4 & 20 & 12 & 3.580 \\
\hline Frugalidad & 11 & 11.08 & 6 & 15 & 11.5 & 2.117 \\
\hline Altruismo & 14 & 13.56 & 5 & 19 & 12 & 3.185 \\
\hline Equidad & 13 & 12.53 & 6 & 15 & 10.5 & 2.412 \\
\hline $\begin{array}{l}\text { Orientación a la } \\
\text { sustentabilidad }\end{array}$ & 38 & 37.16 & 21 & 47 & 34 & 5.792 \\
\hline $\begin{array}{l}\text { Identificación de } \\
\text { oportunidades }\end{array}$ & 13 & 12.53 & 8 & 15 & 11.5 & 1.716 \\
\hline
\end{tabular}

Fuente: Elaboración propia

Como puede observarse en la Tabla 2, la media y la mediana para cada una de las subescalas no difieren mucho. En la mayoría de los casos, para esta muestra, la media es ligeramente menor que la mediana, lo que indica que el conjunto de puntuaciones es simétrico para este grupo encuestado. Las desviaciones estándar de la muestra indican qué tanto se dispersan los datos alrededor de la media (Thorndike y Hagen, 2003). La desviación estándar puede ser utilizada por las 
personas que realicen la encuesta para poder ubicarse dentro de su posición en el grupo con respecto a la media.

Los resultados obtenidos para esta muestra en el total de puntuaciones fueron una media de 110.32 y una desviación estándar de 11.35. En una distribución normal cerca de $68.2 \%$ de los casos se ubicarán dentro del rango +1 y -1 desviaciones estándar de la media, el $95 \%$ entre +2 y -2 desviaciones estándar de la media y casi todos entre +3 y -3 desviaciones estándar de la media (Thorndike y Hagen, 2003).

La distribución de porcentaje, según la desviación estándar permite al participante establecer el estatus que tiene con respecto al grupo. Por ejemplo, una persona que haya obtenido una puntuación de una desviación estándar por arriba de la media sobrepasa al $73 \%$ del grupo; pasando al $50 \%$ que está por debajo de la media y al $23 \%$ que está entre la media +1 desviación estándar.

Los resultados para este estudio indicaron que 58 personas estuvieron entre -1 y 1 desviaciones estándar de la media, 8 personas entre 1 y 2 desviaciones estándar por lo que superaron al $73 \%$ del grupo, 8 personas entre -1 y -2 desviaciones estándar, lo que indica que estuvieron por debajo de la media y 3 personas superaron al 94\% de las personas que alcanzaron más de 2 desviaciones estándar.

La distribución del total de potencial de emprendimiento social se presenta en la figura 1 donde se pueden determinar los porcentajes definidos para esta muestra divididos por rangos. Del total, seis personas se encuentran en un rango de 50 a $59 \%$ de emprendimiento social; 27 personas se ubicaron en el rango de 60 a 69\%; 25 personas en el rango de 70 a 80\%; 24 de 80 a $89 \%$ y de 90 a 100\%, 3 personas. Esto muestra una distribución positivamente asimétrica, en la que la frecuencia tiende hacia las puntuaciones más altas. En este caso, las personas participantes, al ser emprendedores y en una gran mayoría tener un emprendimiento social en desarrollo, se distribuyen en los resultados más altos medidos por el instrumento.

Figura. 1. Distribución de potencial de emprendimiento social.

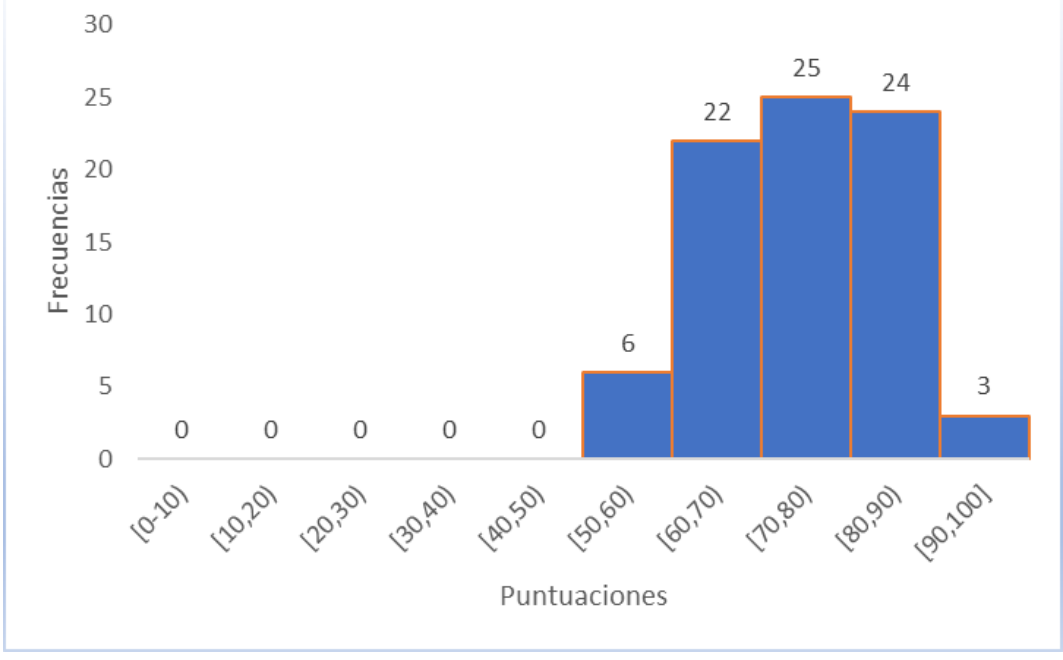


A continuación, se presenta el análisis psicométrico de los resultados obtenidos con el instrumento.

\subsection{Análisis psicométrico del instrumento}

El análisis del instrumento incluye la validez de contenido, de constructo y la confiabilidad del instrumento. La validez de contenido del instrumento se realizó con la revisión de la literatura, donde se encontraron diversos instrumentos que miden constructos relacionados con el emprendimiento social. De esta revisión se seleccionaron los reactivos que fueron utilizados en una prueba piloto.

La confiabilidad de la prueba piloto se determinó utilizando el índice Alfa de Cronbach, para la cual dio un resultado de 0.76 . Por lo que se procedió a eliminar cinco enunciados que hacían que este índice bajara. De los 35 reactivos que conformaban la prueba piloto, se dejaron 30 que presentaron una validez del instrumento de 0.826 .

Una vez aplicado el test al total de la muestra, se procedió a hacer nuevamente el análisis del índice de Cronbach. En esta ocasión se dio como resultado, un índice de fiabilidad para toda la muestra de 0.873 . Este resultado se considera adecuado, ya que si el alfa es mayor a 0.7 , indica consistencia interna del instrumento (Colton, 2007). El análisis del coeficiente alfa, también se realizó para cada una de las subescalas que conforman el constructo potencial de emprendimiento social. En la tabla 3 se muestran los resultados para cada subescala.

Tabla. 3. Índice de Alfa de Cronbach para cada subescala del instrumento

Subescalas

Alfa de Cronbach

Pasión hacia el emprendimiento

0.805

Orientación a la sustentabilidad

Identificación de oportunidades

0.524

Potencial de emprendimiento social

0.873

Fuente: Elaboración propia.

La validez de constructo va a determinar si los reactivos definidos miden adecuadamente la subescala que pretenden medir. Para determinar si esto se cumple en este instrumento, se realizó un análisis de la correlación entre cada uno de los reactivos y la escala a la cual corresponden, con el fin de determinar, cuáles ítems correlacionan más fuerte con los de su misma escala y con su constructo. 
Se pudo determinar que para la escala de pasión hacia el emprendimiento la mayoría de los reactivos tienen una correlación superior al 0.30 , como se muestra en la Tabla 5, lo que indica que sí correlacionan con la escala que están midiendo. Solo tres preguntas están por debajo del 0.30 , que pueden evaluarse en una segunda aplicación del instrumento para determinar si deben ser cambiadas o eliminadas.

Tabla. 5. Análisis de correlación de los reactivos con su escala

Escala

Correlación con el total de elementos de la escala

Pasión hacia el emprendimiento

R1. Desearía estar trabajando cuando no lo estoy .259

R6. Amo realizar esta actividad

R8. Pienso que la actividad que realizo es muy importante para mí

R24. Pienso que la mayor satisfacción de mi vida proviene de mi trabajo en mi emprendimiento

R16.La actividad que me gusta realizar está en armonía con las otras actividades de mi vida

R23. Mi actividad está bien integrada con mi vida

R27.Esta actividad refleja las cualidades que me gustan de mí mismo

R29 Esta actividad me permite vivir una variedad de experiencias

R4. Si pudiera, solo realizaría esta actividad.

.432

R11. Tengo casi una obsesión por esta actividad

.518

R30. He dejado de asistir a actividades personales por estar trabajando

Orientación a la sustentabilidad

R5. Animo a los demás para que reciclen

R10. Leo acerca de temas ambientales

R15. Reciclo productos

R19. Hago saber cuándo dañan el ambiente

R3. Prefiero caminar que viajar en auto

R18.Reutilizo cuadernos y hojas

R28.Prefiero comer en casa que en un restaurante

R7. Participo en colectas de fondos

R12. Regalo ropa usada

R13. Ayudo en tareas a compañeros

R22. Ayudo a personas mayores a cruzar la calle

Identificación de oportunidades

R2. Yo sobrepaso todos los obstáculos para crear una solución a un problema

R9. Me informo con las personas que están trabajando en desarrollar soluciones para problemas en los que estoy interesado.

R17. Mi percepción me permite encontrar soluciones a los problemas 


\section{Conclusiones}

El emprendedor social cuenta con características diferentes a otro tipo de emprendedores, se considera como un agente de cambio, una persona que reconoce una oportunidad dentro de una problemática social (Melián, Campos y Sanchis, 2011). Este trabajo realiza una revisión bibliográfica que permitió observar las características más importantes que debe tener un emprendedor social, con el fin de desarrollar y validar un instrumento que pueda ser utilizado por personas relacionadas con el campo del emprendimiento, que desarrollan capacitaciones, por las incubadoras, dentro de organizaciones que manejen programas de responsabilidad social, como las cooperativas y por los mismos emprendedores, para conocer mejor cuáles son las características que se encuentran en este tipo de emprendedor.

Se encuentra que, en el caso de las empresas de responsabilidad social, como mencionan García Villalobos, Iturrioz del Campo, Mateu Gordon y Palomo Zurdo (2011), buscan mejorar el entorno en el que se desarrollan y deben tomar en cuenta las necesidades de todos los actores involucrados en el proceso productivo, tanto internos como externos, por lo que el perfil de emprendedor social puede ayudarles a detectar mejor las necesidades que estos procesos de involucramiento con la sociedad requieren.

El test de evaluación del potencial en emprendimiento social puede ser utilizado para ayudar a reconocer las características del emprendedor en tres dimensiones relacionadas con este tipo de emprendimiento: pasión hacia el emprendimiento, orientación a la sustentabilidad e identificación de oportunidades. Para determinar la validez del instrumento, se utilizó el Alfa de Cronbach, que para el test general dio como resultado 0.873 , lo cual se considera está dentro de una buena confiabilidad, ya que si es mayor a 0.7 se considera adecuado (Brown, 1980).

Para las tres dimensiones estudiadas, la que se refiere a pasión hacia el emprendimiento se refiere al amor que sienten los emprendedores hacia la actividad que desempeñan (Baum y Locke, 2004) y la cantidad de tiempo y energía que emplean en realizarla (Serrano-Fernández, Boada-Grau, Gil-Ripoll y Vigil Colet, 2017). En este estudio, se utilizaron tres subescalas para medir la pasión hacia el emprendimiento que fueron: apego hacia la actividad, que fue medido por tres ítems $(1,6,8$ y 24$)$, dedicación hacia el emprendimiento, medido por cuatro ítems $(16,23,27$ y 29), y pasión armoniosa, medido por cuatro ítems $(4,11,14$ y 30). La validez de esta escala fue de 0.805 .

Comparando este estudio, con otros encontrados en la literatura se encontró que, en cuanto a la correlación de la escala de pasión hacia el emprendimiento, en algunos enunciados hubo una correlación menor que la presentada en algunos estudios. Por ejemplo, en la versión española de la Escala de la pasión por el trabajo, de Serrano et al (2016), en el enunciado que consulta al participante si la actividad que realiza está en armonía con las otras actividades de su vida, representó en este estudio una correlación de 0.523 y en la versión española de 0.647. En el enunciado que consulta la dedicación hacia el emprendimiento la correlación para el instrumento de este estudio fue de 0.518 y la de Serrano et al (2016) fue de 0.869. 
La segunda dimensión que se midió fue la orientación a la sustentabilidad, que es la convicción que tiene el emprendedor de hacer que su negocio se desarrolle ecológicamente y sea socialmente responsable (DiVito y Bohnsack, 2017). Para este estudio se midieron cuatro subescalas: la conducta pro-ecológica (ítem 5,10,15 y 19), la frugalidad (ítem 3,18 y 28), el altruismo (ítem 7,12,13 y 22) y la equidad (ítem 20,21,26). La validez de esta escala fue de 0.831 . Comparando los resultados con el estudio de Corral Verdugo et al (2016), se obtiene que para las subescalas de altruismo se obtuvo un alfa de Cronbach de 0.78 y en este estudio 0.640 , en el de conducta pro-ecológica 0.82 en Corral et al (2016) y en este estudio 0.787.

La identificación de oportunidades consiste según Vogel (2016) en reconocer e interpretar la información que recibe el emprendedor para determinar si valora el desarrollo de esa actividad. En este apartado, se recomienda analizar más a fondo las respuestas para la pregunta abierta, considerando la calidad de las mismas. En este estudio se obtuvieron más de 176 soluciones al problema planteado.

Dentro de los alcances y limitaciones del presente trabajo se considera importante indicar que si bien el emprendedor social tiene características que lo diferencian del resto de empresarios, como que su principal objetivo no es la búsqueda de lucro sino la posibilidad de generar propuestas de valor para la sociedad, se eligieron tres escalas que según la literatura encontrada pudieran ser importantes para iniciar con este tipo de emprendimientos, por lo que se sugiere que para futuras investigaciones se continúen explorando otras características de este tipo de emprendedor como sus competencias de comunicación, trabajo en equipo, entre otras.

Una limitación encontrada en el procedimiento de aplicación fue el acceso a las personas emprendedoras, debido a que se encontraban en diferentes lugares y que el instrumento se envió por correo electrónico, por lo que el nivel de respuesta se limitó a un grupo que estuvo dispuesto a participar. Para futuras investigaciones, se recomienda analizar las relaciones que existen entre las diferentes subescalas medidas en este instrumento.

Debido al límite de extensión de esta publicación y al enfoque, queda pendiente la exposición de resultados derivados de los análisis cualitativos realizados a los reactivos de preguntas abiertas que permitan explorar con mayor profundidad los tipos de soluciones que los emprendedores implementan ante problemas sociales. Estos datos pueden ayudar a conocer la creatividad e innovación presente en el emprendedor social y conocer sus observaciones a través de este instrumento.

El test de potencial en emprendimiento social es una herramienta que puede ser utilizada para medir las diferentes características de un emprendedor social. Su utilización permitirá que las personas que toman decisiones respecto a procesos de formación y capacitación de este tipo de emprendedores puedan desarrollar actividades relacionadas con sus características. Además, permite al mismo emprendedor conocer cuáles son sus particularidades y poder desarrollar dentro de sus empresas, actividades que busquen dar soluciones a los problemas que afronta la sociedad. 


\section{Referencias bibliográficas}

Álvarez, S. y Barney, J. (2014) Entrepreneurial opportunities and poverty alleviation. Entrepreneurship: Theory \& Practice, № 38, Vol. 1, 159-184. https://doi.org/10.1016/j.protcy.2015.07.044

Baum, J. y Locke, E. (2004) The relationship of entrepreneurial traits, skill, and motivation to subsequent venture growth. Journal of Applied Psychology, $\mathrm{N}^{\circ}$ 89, Vol. 4, 587-598. DOI: 10.1037/0021-9010.89.4.587. Disponible en:

http://citeseerx.ist.psu.edu/viewdoc/download?doi=10.1.1.457.7703\&rep=rep1\&type=pdf

Benito Hernández, S. (2009) El papel de las microempresas en el desarrollo económico regional: las redes de cooperación empresarial en España. REVESCO. Revista de Estudios Cooperativos, Tercer Cuatrimestre, $\mathrm{N}^{\mathrm{o}}$ 99, pp. 31-59. DOI: 10.5209/REVE.19591.

Biraglia, A. y Kadile, V. (2017) The role of entrepreneurial passion and creativity in developing entrepreneurial intentions: Insights from American Homebrewers. Journal of Small Business Management, No 55, pp. 170-188. DOI: 10.1111/jsbm.12242.

Boeve-De Pauw, J. y Van Petegem, P. (2017) Because my friends insist or because it makes sense? Adolescents' Motivation towards the Environment. Sustainability, No 9, Vol. 5, 750. DOI: $10.3390 / \mathrm{su} 9050750$.

Brinkerhoff, P. (2000) Social Entrepreneurship: The art of mision-based venture development. NY: Wiley.

Brown, F. (1980) Principios de la medición en psicología y educación. México: Manual Moderno.

Cardon, M., Glauser, M. y Murnieks, C.J. (2017) Passion for what? Expanding the domains of entrepreneurial passion. Journal of Business Venturing Insights, $\mathrm{N}^{\mathrm{o}}$ 8, pp. 24-32. Disponible en: http://digitalcommons.pace.edu/cgi/viewcontent.cgi?article=1002\&context=wilson

Chen, H., Chen, F., Huand, X., Long, R. y Li, W. (2017) Are individuals' environmental behavior always consistent? An analysis based on spatial difference. Resources, Conservation and Recycling, $\mathrm{N}^{\circ} 125$, pp. 25-36.

Ciruela Lorenzo, A., Cuadrado Serrán, M. y Plaza Angulo, J. (2016) La economía social como alternativa de autoempleo. El perfil del emprendedor/a en las sociedades laborales andaluzas. REVESCO. Revista de Estudios Cooperativos, Tercer Cuatrimestre, $\mathrm{N}^{\circ} 122$, pp. 59-85. DOI: 10.5209/REVE.2016.v122.52019.

Colton, D. y Covert, R. (2007) Designing and constructing instruments for social research and evaluation. New York: Willey.

Corral-Verdugo, V., Tapia, C., Frías, M., Fraijo, B. y González, D. (2009) Orientación a la Sostenibilidad como base para el Comportamiento Pro-Social y Pro-Ecológico. Medio Ambiente y Comportamiento Humano, $N^{o}$ 10, Vol. 3, pp. 195-215. Disponible en: https://mach.webs.ull.es/PDFS/Vol10 3/Vol10_3 b.pdf

Corral-Verdugo, V., Tapia-Fonllem, C. y Ortiz-Valdez, A. (2014) On the Relationship Between Character Strengths and Sustainable Behavior. Environment and Behavior, $\mathrm{N}^{\mathrm{o}}$ 1, 25. https://doi.org/10.1177/0013916514530718

De Clercq, D. y Belausteguigoitia, I. (2017) Overcoming the dark side of task conflict: Buffering roles of transformational leadership, tenacity, and passion for work. European Management Journal, No 35, pp. 78-90. https://doi.org/10.1016/j.emj.2016.06.008

Divito, L. y Bohnsack, R. (2017) Entrepreneurial orientation and its effect on sustainability decision tradeoffs: The case of sustainable fashion firms. Journal of Business Venturing, $\mathrm{N}^{\mathrm{o}}$ 32, pp. 569-587. https://doi.org/10.1016/j.jbusvent.2017.05.002 
Gagné, M. (2014) The Oxford Hanbook of work engagement, motivation, and selfdetermination theory. New York: Oxford University Press.

Garcia, J.C.; Iturrioz, J.; Mateu, J.L.; Palomo, R. (2011) La percepción sobre la responsabilidad social en las sociedades cooperativas de trabajo asociado y las sociedades laborales: un análisis en el ámbito de la ciudad Madrid, REVESCO. Revista de Estudios Cooperativos, Primer Cuatrimestre, № 104, pp. 102-124.

González, M., Husted, B. y Aigner, D. (2017) Opportunity discovery and creation in social entrepreneurship: An exploratory study in Mexico. Journal of Business Research. https://doi.org/10.1016/j.jbusres.2016.10.032

Herrera-Mendoza, K. (2016). Attitudes and ecological behavior of college students. Opcion, $\mathrm{N}^{\mathrm{o}} 32$, Vol. 13, pp. 456-477. Disponible en: http://www.redalyc.org/articulo.oa?id=31048483023

Hossain, S., Saleh, M.A. y Drennan, J. (2017) A critical appraisal of the social entrepreneurship paradigm in an international setting: a proposed conceptual framework. International Entrepreneurship and Management Journal, $\mathrm{N}^{\mathrm{o}}$ 13, Vol. 2, pp. 347-368. DOI: 10.1007/s11365-016-0400-0.

Iturrioz, J. y Dopacio, I. (2009) La Responsabilidad Social en las sociedades cooperativas: una perspectiva económico financiera. Cuadernos de Estudios Empresariales, $\mathrm{N}^{\mathrm{o}} 19$, pp. 153-173.

Kaiser, F., Carabias-Hütter, V. y Scheuthle, H. (2005) The motivational and instantaneous behavior effects of contexts: steps toward a theory of goal-directed behavior. Journal of Applied Social Psychology, $\mathrm{N}^{\mathrm{o}}$ 35, Vol. 10, pp. 2076-2093. Disponible en: https://pd.zhaw.ch/publikation/upload/1145.pdf

Kliksberg, B. (2011) Emprendedores sociales. los que hacen la diferencia. Buenos Aires: Temas.

Kuckertz, A. y Wagner, M. (2010) The influence of sustainability orientation on entrepreneurial intentions -Investigating the role of business experience. Journal of Business Venturing, № 25, pp. 524-539. Disponible en: http://www.redalyc.org/pdf/847/84725699005.pdf

Martin, F. y Binder, J. (2015) Sustainable Entrepreneurship: A Convergent Process Model. Business Strategy and the Environment, $\mathrm{N}^{\mathrm{o}}$ 26, Vol. 1, pp. 1-17. DOI: 10.1002/bse.1887.

Melián Navarro, A., y Campos Climent, V. (2010). Emprendedurismo y economía social como mecanismos de inserción sociolaboral en tiempos de crisis. REVESCO. Revista de Estudios Cooperativos, No 100, pp. 43-67. DOI: 10.5209/REVE.19552.

Melián Navarro, A., Campos Climent, V. y Sanchis Palacio, J. (2011) Emprendimiento social y empresas de inserción en España. Aplicación del método Delphi para la determinación del perfil del emprendedor y las empresas sociales creadas por emprendedores. REVESCO. Revista de Estudios Cooperativos, Tercer Cuatrimestre, $\mathrm{N}^{\mathrm{o}}$ 106, pp. 150-172. DOI: 10.5209/rev_REVE. 2011.v106.37377.

Roxas, B., y Chadee, D. (2012) Environmental sustainability orientation and financial resources of small manufacturing firms in the philippines. Social Responsibility Journal, № 8, Vol. 2, pp. 208-226.

Sahai, R. y Frese, M. (2017) If you have a hammer, you only look for nails: the relationship between the Einstellung effect and business opportunity identification. Entrepreneurship Theory and Practice. DOI: 10.1111/1540-8520.00003.

Serrano, M.J., Boada-Grau, J., Gil-Ripoll, C. y Vigil-Colle, A. (2017) Spanish adaptation of the Passion Toward Work Scale. Anales de Psicología, № 33, Vol. 2, pp. 403-410. DOI: http://dx.doi.org/10.6018/analesps.33.2.240521. 
Surie, G. (2017) Creating the innovation ecosystem for renewable energy via social entrepreneurship: Insights from India. Technological Forecasting and Social Change, $\mathrm{N}^{\mathrm{o}}$ 121, pp. 184-195. DOI: 10.1111/jsbm.12346.

Tapia-Fonllem, C., Fraijo-Sing, B., Corral-Verdugo, V. y Ortiz Valdez, A. (2017) Education for Sustainable Development in Higher Education Institutions. SAGE Open, $\mathrm{N}^{\mathrm{o}}$ 7, Vol. 1, pp. 1-15.

Tapia-Fonllem, C., Corral-Verdugo, V., Fraijo-Sing, B. y Durón-Ramos, M.F. (2013) Assessing sustainable behavior and its correlates: A measure of pro-ecological, frugal, altruistic and equitable actions. Sustainability, $\mathrm{N}^{\mathrm{o}}$ 5, Vol. 2, pp. 711-723. DOI: 10.3390/su5020711.

Thorndike, R. y Hagen, E. (2003) Medición y evaluación en psicología y educación. México: Trillas.

Vallerand, R.J. (2010) Chapter Three: On passion for life activities: The dualistic model of passion. Advances in Experimental Social Psychology, $\mathrm{N}^{\circ}$ 42, pp. 97-193. DOI: 10.1016/S0065-2601(10)42003-1.

Vogel, P. (2016) From Venture Idea to Venture Opportunity. Entrepreneurship Theory and Practice. DOI: 10.1111/etap.12234.

Welter, C., Mauer, R. y Wuebker, R. (2016) Bridging Behavioral Models and Theoretical Concepts: Effectuation and Bricolage in the Opportunity Creation Framework. Strategic Entrepreneurship Journal, No 10, Vol. 1, pp. 5-20. DOI: 10.1002/se.

Wry. T. y York, J. (2017) An identity-based approach to social Enterprise. Academy of Management Review, $\mathrm{N}^{\circ}$ 42, Vol. 3, pp. 437-460. Disponible en: http://www.effectuation.org/wp-content/uploads/2017/05/An-Identity-Based-Approach$\underline{1 . p d f}$

Yunus, M. (2010) Empresas para todos. México: Norma. 


\section{Anexo 1}

Fecha:

\section{Test de evaluación del potencial en emprendimiento social}

Estimado participante:

En este cuestionario le presentamos una serie de preguntas relacionadas con las actitudes y comportamientos que presenta una persona que realiza un emprendimiento social, con el fin de conocer su perfil y determinar el potencial que tienen los participantes para realizar este tipo de actividades. Los datos que nos suministre se manejarán de manera confidencial. Agradecemos su participación en el estudio pues nos dará datos importantes para generar oportunidades de capacitación para este sector emprendedor. Responda de la forma más honesta posible, no hay respuestas buenas ni malas, lo que nos interesa es conocer su opinión.

\section{Favor complete la información que se solicita: \\ I Parte: Datos de identificación}

Edad:

Género:

Lugar de $\overline{\text { residencia: }}$

Último grado académico obtenido:

Trabaja actualmente:

¿Qué tipo de emprendimiento tiene en este momento?

¿Hace cuánto tiempo lo tiene?

¿Definiría su emprendimiento como social?

\section{Parte. Desarrollo de nuevas ideas}

En esta parte esperamos que usted colabore con nosotros proponiendo ideas que den solución al problema planteado a continuación. Puede escribir todas las que se le vengan a la mente. Solo debe enunciarlas no explicarlas o elaborarlas.

Usted está viendo las noticias y escucha que el problema del manejo de residuos es un grave problema para las grandes ciudades y la población mundial y que la solución es reusar, reducir y reciclar, ya algunos emprendedores están proponiendo ideas de negocio para minimizar esta situación ¿Cuáles ideas de negocio vienen a su mente? Haga una lista de todas las ideas de negocio posibles, las ideas pueden estar o no relacionadas con su área actual o sus intereses. 
III Parte: Actitudes y comportamientos hacia el emprendimiento En esta parte estamos midiendo la actitud de los participantes hacia la actividad que realizan. Agradecemos conocer su experiencia contestando el cuestionario según las siguientes categorías:

\begin{tabular}{|c|c|c|c|c|c|c|c|c|c|}
\hline & 1 & 2 & 3 & 4 & \multicolumn{5}{|c|}{5} \\
\hline \multicolumn{2}{|r|}{ Nunca } & Casi nunca & Algunas veces & Casi siempre & \multicolumn{5}{|c|}{ Siempre } \\
\hline & & & & & 1 & 2 & 3 & 4 & 5 \\
\hline 1 & \multicolumn{4}{|c|}{ Desearía estar trabajando cuando no lo estoy } & & & & & \\
\hline 2 & \multicolumn{4}{|c|}{ Sobrepaso todos los obstáculos para crear una solución a un problema } & & & & & \\
\hline 3 & \multicolumn{4}{|c|}{ Prefiero caminar que viajar en auto } & & & & & \\
\hline 4 & \multicolumn{4}{|c|}{ Si pudiera, solo realizaría esta actividad. } & & & & & \\
\hline 5 & \multicolumn{4}{|c|}{ Animo a los demás para que reciclen } & & & & & \\
\hline 6 & \multicolumn{4}{|c|}{ Amo realizar esta actividad } & & & & & \\
\hline 7 & \multicolumn{4}{|c|}{ Participo en colectas de fondos } & & & & & \\
\hline 8 & \multicolumn{4}{|c|}{ Pienso que la actividad que realizo es muy importante para mí } & & & & & \\
\hline 9 & \multicolumn{4}{|c|}{$\begin{array}{l}\text { Me informo con las personas que están trabajando en desarrollar } \\
\text { soluciones para problemas en los que estoy interesado }\end{array}$} & & & & & \\
\hline 10 & \multicolumn{4}{|c|}{ Leo acerca de temas ambientales } & & & & & \\
\hline 11 & \multicolumn{4}{|c|}{ Tengo casi una obsesión por esta actividad } & & & & & \\
\hline 12 & \multicolumn{4}{|c|}{ Regalo ropa usada } & & & & & \\
\hline 13 & \multicolumn{4}{|c|}{ Ayudo en tareas a compañeros } & & & & & \\
\hline 14 & \multicolumn{4}{|c|}{$\begin{array}{l}\text { Cuando converso con otras personas les digo que debería estar } \\
\text { trabajando }\end{array}$} & & & & & \\
\hline 15 & \multicolumn{4}{|c|}{ Reciclo productos } & & & & & \\
\hline 16 & \multicolumn{4}{|c|}{$\begin{array}{l}\text { La actividad que me gusta realizar está en armonía con las otras } \\
\text { actividades de mi vida }\end{array}$} & & & & & \\
\hline 17 & \multicolumn{4}{|c|}{ Mi percepción me permite encontrar soluciones a los problemas } & & & & & \\
\hline 18 & \multicolumn{4}{|c|}{ Reutilizo cuadernos y hojas } & & & & & \\
\hline 19 & \multicolumn{4}{|c|}{ Hago saber cuándo dañan el ambiente } & & & & & \\
\hline 20 & \multicolumn{4}{|c|}{ Pienso que los niños tienen mismo derecho que los adultos } & & & & & \\
\hline 21 & \multicolumn{4}{|c|}{$\begin{array}{l}\text { Pienso que el hombre y la mujer tienen las mismas obligaciones en } \\
\text { casa }\end{array}$} & & & & & \\
\hline 22 & \multicolumn{4}{|c|}{ Ayudo a personas mayores a cruzar calle } & & & & & \\
\hline 23 & \multicolumn{4}{|c|}{ Mi actividad está bien integrada con mi vida } & & & & & \\
\hline 24 & \multicolumn{4}{|c|}{$\begin{array}{l}\text { Pienso que la mayor satisfacción de mi vida proviene de mi trabajo en } \\
\text { mi emprendimiento }\end{array}$} & & & & & \\
\hline 25 & \multicolumn{4}{|c|}{ Despierto pensando en cosas del trabajo } & & & & & \\
\hline 26 & Los rec & aturales deben & rtirse equitativame & & & & & & \\
\hline 27 & Esta act & refleja las cuali & es que me gustan c & mí mismo & & & & & \\
\hline 28 & Prefier & en casa que en & restaurante & & & & & & \\
\hline 29 & Esta act & me permite viv & a variedad de exp & encias & & & & & \\
\hline 30 & He deja & sistir a activida & personales por est & trabajando & & & & & \\
\hline
\end{tabular}

